\title{
Metabolismo de los lípidos durante el embarazo
}

\author{
José Henry Osorio O.*
}

Recibido: Marzo 14 / 2000

Revisado: Abril 2 / 2000

Aceptado: Junio 9/ 2000

\section{RESUMEN}

El flujo neto de nutrientes, electrolitos, vitaminas, y otros compuestos de la madre a el feto es necesario para asegurar un suministro constante para el metabolismo energético fetal así como para el crecimiento y diferenciación de tejidos. En la formación de sistemas fetales como el nervioso y en el balance de requerimientos extra de energía en el embarazo avanzado es indispensable la utilización de los lípidos. En la presente revisión podemos recordar los mecanismos básicos y la secuencia de eventos metabólicos relacionados con estos durante la gestación.

PALABRAS CLAVES: Embarazo, Metabolismo, Lípidos.

\section{SUMMARY}

The net flux of nutrients, electrolytes, vitamins, and other compounds from the mother to the fetus is needed to secure a constant supply for fetal energy metabolism as well as tissue growth and differentiation. For fetal systems formation like central nervous system and for the mechanisms and the metabolic events sequence related to them during the pregnancy.

\section{KEY WORDS: Pregnancy, Metabolism, Lipids.}

\section{Introducción}

El balance energético a través de la gestación, depende de la estrecha relación madre-placenta-feto mediada por hormonas, garantizando la conservación de energía y el adecuado aprovechamiento de nutrientes, siendo los lípidos como las demás biomoléculas, de capital importancia en el proceso de formación del nuevo bebé.

Los lípidos son biomoléculas donde predominan las cadenas hidrocarbonadas (-CH2-CH2-CH2-) en su estructura, presentado por eso naturaleza hidrofílica, lo que los hace insolubles o muy poco solubles en agua, y solubles en solventes no polares como éter, cloroformo o benceno.

Sus funciones generales son el almacenamiento de combustible metabólico, el transporte de combustible metabólico, como aislante en buena parte del organismo con fines de protección, y como componente estructural de membranas celulares y subcelulares (1).

En la presente revisión, hacemos énfasis en su metabolismo, por lo cual se hace necesario recordar una serie de características de este gran grupo de compuestos, antes de avanzar describiendo procesos de degradación o síntesis. Temas como el metabolismo de carbohidratos y lípidos, gasto energético y balance de nitrógeno, cambios metabólico-hormonales, y bases funcionales placentarias han sido revisadas anteriormente (2).

Profesor Asociado. Director de la línea de Investigación en Bioquímica y Salud. Universidad de Caldas. Manizales. Colombia. Child Health Departament. Faculty of Medicine. University of Newcastle upon Tyne. England
Son utilizadas en la presente revisión, siglas internacionales para diferentes compuestos y enfermedades.

\section{Definición de términos $(3,4,5,6)$}

Acidos grasas: Compuestos insolubles en agua, con un grupo carboxilo al final de la cadena, saturados (sin dobles enlaces en la cadena) o insaturados (con dobles enlaces en la cadena), los cuales sirven al organismo como fuente de energía, y como precursores para la síntesis de otras moléculas como los triacilgliceroles (TAG) o triesteres de glicerol unidos a tres ácidos grasos, siendo la forma preferida de almacenamiento de energía, y los cuerpos cetónicos, formados de ácidos grasos y carbohidratos, solubles en agua (acetona, acetato y $\beta$ hidroxibutirato) utilizados como fuente de energía.

Fosfolípidos: Lípidos que contienen fósforo, unidos a un esqueleto de glicerol, (fosfoglicéridos y esfingosina), son el mayor componente de las membranas celulares con altas concentraciones en órganos glandulares y plasma sanguíneo. Entre los fosfoglicéridos o triesteres de glicerol-3-fosfato se cuentan: fosfatidilcolina (lecitina), fosfatidiletanolamina (una cefalina), fosfatidilserina (una cefalina), fosfatidilinositol y cardiolipina.

Esfingolípidos: Derivados todos de la esfigosina, presentes en casi todos los tejidos del organismo, con una gran concentración en sistema nervioso central (CNS de central nervous system), especialmente en la materia blanca y los cuales incluyen la esfingomielina (formados por ceramida y citidina difosfato-colina) y glicofosfolípidos (cerebrósidos, sulfátidos, globósidos y gangliósidos). 
Eicosanoides: Productos del metabolismo del ácido araquidónico (ácido graso insaturado de 20 átomos de carbono), con funciones moduladoras de la actividad fisiológica, dentro de los cuales se cuentan las prostaglandinas (análogos del ácido prostanóico) tromboxanos (análogos del ácido prostanóico pero con anillo en su estructura que contiene oxígeno), los ácidos hidroperoxieicosatetraenóicos (HPETE) (derivados del ácido araquidónico sin anillo en su estructura) y los leucotrienos (LT) (formados de HPETE pero con tres dobles enlaces conjugados).

Esteroides: Lípidos que contienen cuatro anillos de carbono fusionados conformando el nucleo esteroidal (cilcopentanoperhidrofenantreno), subdivididos en: esteroles, colesterol y ácidos biliares, los cuales actúan como componentes de membrana y moduladores de la actividad fisiológica.

Lipoproteínas: Complejos de proteína y lípidos, ensamblados juntos mediante enlaces no covalentes, siendo las más importantes las lipoproteínas plasmáticas, cuya función es el transporte delípidos, de las cuales con base en la densidad (d), determinada por la tasa de flotación a una centrifugación de $1.063 \mathrm{~d}$ en una solución de cloruro de sodio, se distinguen 5 fracciones conocidas como: lipoproteínas de alta densidad (HDL de high-density lipoproteins), lipoproteínas de baja densidad (LDL, de low-density lipoprotein), lipoproteínas de densidad intermedia (IDL de intermediate-density lipoprotein), lipoproteínas de muy baja densidad (VLDL de very low- density lipoproteins) y quilomicrones.

\section{Requerimiento energético cubierto por el metabolismo graso}

En condiciones normales, la proteína es requerida con propósitos estructurales solamente y no para actividad. Los carbohidratos como fuente de combustible son requeridos por el cerebro materno, el cual utiliza glucosa, y por el feto, que oxida casi exclusivamente carbohidratos.

Solo una pequeña parte de carbohidrato es requerida como material estructural del cerebro, cartílago y tejido conectivo.

Además de los requerimientos grasos para el trabajo muscular, existe la necesidad de ácidos grasos insaturados de cadena larga, los que son escenciales como componentes de las membranas celulares y subcelulares y como precursores de metabolitos biológicamente activos los cuales incluyen prostaglandinas, prostaciclinas, tromboxanos, y leucotrienos (7).

Otro papel desempeñado por las grasas es el del transporte y absorción de vitaminas liposolubles y otras sustancias.

Las demandas adicionales de energía relacionadas con el embarazo se deben al volumen de proteína y grasa que debe ser acumulada por el feto y la madre, y la adición al metabolismo en el cual los nuevos tejidos incurren.

Los gastos de energía extra en los cuales incurre el sistema materno, tienen que ver con el esfuerzo en corazón, pulmones, y riñones para el mantenimiento de los nuevos tejidos, y el costo del mantenimiento de la temperatura corporal más alta en el embarazo, aunque se sabe, que la termogénesis postprandial, está reducida en las mujeres bien alimentadas durante los últimas semanas de gestación, algo que es todavía materia de investigación (8).
Durante el último $3 / 4$ del embarazo, el requerimento de energía extra atribuible al robustecimiento de los nuevos tejidos puede llegar en promedio a ser un máximo de $240 \mathrm{Kcal}$ (IMJ) diariamente, y el consumo de oxígeno adicional es estimado en un promedio de $150 \mathrm{Kcal}(0.6 \mathrm{MJ})$ al dia, sumando un total de $390 \mathrm{Kcal}(1.6 \mathrm{MJ})$ en promedio diariamente (9).

Durante el $1 / 4$ final del embarazo, el creecimiento de los tejidos fetales se incrementa rápidamente, pero la tasa de almacenamiento de lípidos por parte de la madre está notablemente reducida. La energía representada por los incrementos diarios de nuevos tejidos es solo de cerca de 65 $\mathrm{Kcal}(0.3 \mathrm{MJ})$, en promedio. El promedio total de adición a los requerimientos de energía durante las últimas semanas de la gestación, es de cerca de $300 \mathrm{Kcal}$ (1.3 MJ) por día (10).

Los requerimientos específicos de energía son los mayores durante los 2/4 medios de la gestación, cuando grandes cantidades de grasa materna están siendo acumuladas como banco de energía. Durante el cuarto final, tal almacenamiento prácticamente se detiene y la mayor parte de la energía requerida para la formación de nuevos tejidos es la debida al crecimiento del feto, el cual se acompaña de considerable elevación en el consumo de oxígeno. El efecto final es la distribución de las adiciones a las necesidades de (energía total en las últimas 3/4 partes de la gestación sin un incremento en la demanda en las últimas semanas.

Aunque el costo de la energía para la actividad está aumentado en las últimas etapas de la gestación, alguna reducción en la cantidad de actividad suele ser lo usual, sin necesidad de un aporte extra en la dieta, esto parece ser una característica fisiológica más que un cambio consciente de conducta en búsqueda de descansó (11).

\section{Flujo materno de lípidos $y$ embarazo}

El proceso de adaptación del sistema materno al metabolismo lipídico en el embarazo consta de un incremento en el almacenamiento de lípidos en un período temprano (12), incremento en la absorción intestinal de nutrientes acompañado por aumento en la demanda de los mismos (13) y un importante flujo, particularmente de EFA y LC-PUFA de la madre al feto (14).

Aunque la glucosa es el nutriente primario para el feto, existe un gran aumento de TAG como tejido adiposo durante el último trimestre (15). Además son necesarios ácidos grasos poliinsaturados de cadena larga (LC-PUFA de long-chain polyunsaturated fatty acids) particularmente araquidónico (AA.20:4n-6) y docosahexaenoico (DHA,22:6n-3) para la formación de membranas en todos los órganos, especialmente en el cerebro (16), el araquidónico es también un precursor importante de las moléculas lipídicas de senalización. Los LCPUFA son sintetizados en cantidad insuficiente por el feto y la placenta aparentemente no posee la actividad $\alpha-5$ y $\alpha-6$ desaturasa para convertir ácidos grasos esenciales (EFA de essential fatty acids) en LC-PUFA $(17,18)$, es por eso que existe un mecanismo preferencial para síntesis y transferencia de estos ácidos grasos hacia la placenta y el feto.

El contenido total de fosfolípidos en plasma está incrementado durante la gestación, acompañado por un incremento en las concentraciones de ácido linoléico, AA y DHA, pero están más incrementados aun los ácidos grasos 
saturados y monoinsaturados, de tal forma que el estatus escencial ácido graso (n-3 + n-6)/(n-7+n-9) de ácidos grasos en los fosfolípidos del plasma disminuye significativamente, por lo cual algunos autores postulan el ambarazo asociado con déficit materno de EFA (19), sin embargo otros estudios muestran un buen estatus de EFA al comienzo de la gestación, el cual no previene la caída de este índice en etapas posteriores (20). La importancia de DHA radica en su papel definitivo en los procesos visuales, con incremento de la cantidad de DHA en la retina humana con la maduración fetal (21), siendo postulado, por lo tanto, como esencial para el crecimiento del cerebro y la retina $(22,23,24)$. Además la formación activa de estructuras sinápticas y arborizaciones dendítricas con una rápida multiplicación de espinas dendítricas, la cual se incrementa rápidamente entre la semana 31 de la gestación y el nacimiento (25), estos cambios son detectados morfológica y bioquímicamente $(26,27)$. Otros ácidos grasos parecen ser importantes durante el embarazo, ya que han sido encontrados niveles disminuidos de ácido linoléico (18:2n-6, LA) en hipertensión inducida por el embarazo $(28,29)$ y retardo en el crecimiento intrauterino (30).

Por eso ha sido postulada la suplementación con LC- PUFA especialmente DHA en la dieta, lo cual puede ser logrado incrementando el consumo de pescado en la alimentación, por ser rico en -3 LC- PUFA, logrando con un aumento de 10 veces en el consumo de pescado un pequeño incremento en los DHA en las membranas de los eritrocitos de la madre y el feto y una concomitante disminución de AA (31), favoreciendo este hallazgo el concepto de que un incremento en $n-3$ ácidos graso s tiene efectos saludables en el embarazo incluyendo disminucion del riesgo de labor pretérmino e hipe.rtensión inducida por el embarazo $(32,33,34,35,36))$, aunque se hace necesaria mucha más investigación en la relación entre nutrición y presentación de estos problemas.

\section{Movilización de reservas maternas}

La grasa corporal materna acumulada durante el primer trimestre de la gestación, permanece estable durante todo el embarazo (37) a expensas de un continuo e intenso aporte de glucosa, aminoácidos y otras sustancias a el feto, estando la lipólisis en el tejido adiposo mejorada en el último trimestre causando un incremento en la circulación de ácidos grasos libres (FFA de Free Fatty Acids) y glicerol (38).

En el hígado materno, los FFA son activados y usados para ser esterificados y formar glicéridos o para ser degra- dados a acetyl-CoA y síntesis de cuerpos cetónicos a través de la $\beta$ oxidación, mientras que el glicerol en forma de alfa glicerol fosfato es usado para la síntesis de glicéridos o para gluconeogénesis.

En estados de ayuno, la lipólisisde tejido adiposo y el uso metabólico de sus productos, están aumentados en la mujer embarazada (39) mediante la denominada "hambre acelerada" ( accelerated starvation), observándose entonces niveles circulantes mas altos de FFA cuerpos cetónicos y glicerol en estados avanzados de gestación en las mismas condiciones de ayuno, comparados con no embarazadas, y los niveles de FFA en el plasma fetal permanecen bajos demostrando las dificultades de estos compuestos para atravezar la barrera placentaria, mientras que los cuerpos cetónicos, producto de la degradacion de FFA en

la $\beta$-oxidación, pueden cruzar libremente dicha barrera observándose la misma concentración tanto en plasma fetal como en plasma materno (40).

Los cuerpos cetónicos entonces, pueden ser utilizados como combustible por el feto, incluso para la síntesis de lípidos cerebrales, ayudando a garantizar el desarrollo fetal normal, aún en condiciones de ayuno o intensa hipoglicemia materna (41).

Por el contrario, los niveles de glicerol en plasma fetal están disminuidos como consecuencia de una capacidad de transporte limitada a través de la placenta (42), pero dado que el glicerol puede ser convertido en glucosa materna, de esta forma el feto puede beneficiarse indirectamente de altos niveles de glicerol recibiendo glucosa efectivamente. (43).

De esta manera en etapas avanzadas de la gestación, la mujer proveee substratos necesarios al feto aún en el ayuno, mientras que su homeostasis metabólica se mantiene a expensas del metabolismo de los lípidos en sus propios tejidos.

Uno de los principales hallazgos durante el tercer trimestre del embarazo es la llamada hipertrigliceridemia del tercer trimestre, la cual tiene como función principal, la preparación de la madre para la futura lactancia post-parto, los principales eventos metabólicos implicados en el proceso son:

1.- Niveles de todas las lipoproteínas elevadas durante el segundo trimestre de la gestación (44).

2.- Lipoproteínas ricas en triglicéridos (quilomicromes y VLDL) se encuentran circulando en mayor cantidad (45).

3.- Incremento en la producción endógena de triglicéridos (46).

4.- Aumento en la transmisión de FFA al hígado proveniente de la lipólisis en tejido adiposo (47).

5.- Tendencia al apetito aumentado con reducción del tránsito gastrointestinal, y una absorción intestinal sin cambios lo que conlleva a un mayor aporte de triglicéridos de la dieta a la circulación materna (48).

6.- Disminución de la actividad de lipoproteína lipasa (LPL de lipoprotein lipase) en tejido adiposo (49).

Además antes del parto se incrementa la actividad de la LPL en glándula mamaria, probablemente desencadenado por la liberación de prolactina (50), con facilidad de captación de lípidos aumentada para la glándula y reducción de triglicéridos circulantes, con reducción de las VLDL circulantes aun estando bajos los niveles de LDL en tejido adiposo, favoreciendo todos estos factores la producción de leche, (51).

\section{Placenta y movimiento lipídico}

A través de la gestación FFA son requeridos por el feto y la placenta para la síntesis de lípidos, incluyendo fosfolípidos, glicolIpídos, esfingolípidos, ésteres de colesterol y TAG (52).

Muchos de esos lípidos forman importantes componentes de membranas celulares, jugando por lo tanto, un papel importante en el proceso de crecimiento placentario y fetal, en particular del sistema nervioso fetal (52). El feto humano contiene el. $0.5 \%$ de grasa a mediados de la gestación, iniciando el proceso de acumulación de grasa blanca, llegando a las 28 semanas a contener un $3.5 \%$ 
Figura 0014i01

PRINCIPALES RUTAS DEL METABOLISMO DE LOS LIPIDOS EN EL EMBARAZO E

HYPERTRIGLICERIDEMIA MATERNA EN EL TERCER TRIMESTRE DEL EMBARAZO COMO PREAMBULO A LA LACTANCIA

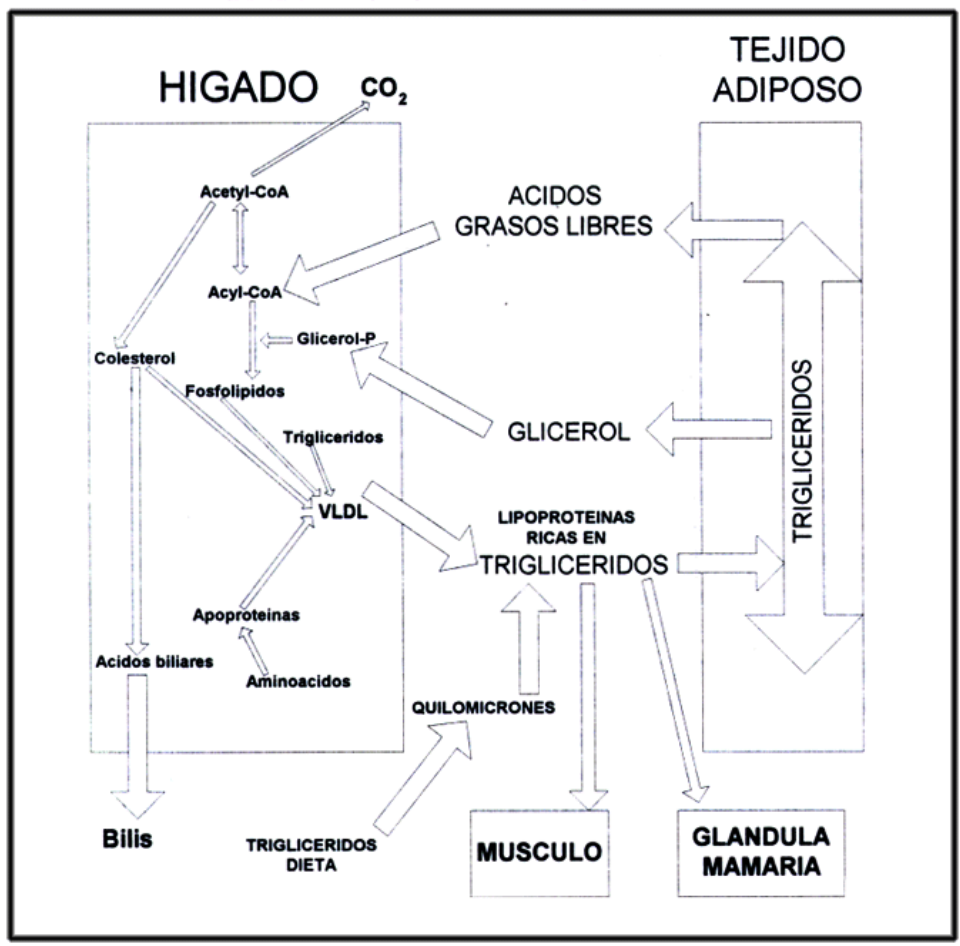

aproximadamente y a las 34 semanas entre 7 y $8 \%$, con valores para el recién nacido de $16 \%$ aproximadamente (54). Estudios in vitro con tejidos placentarios humanos demuestran que aproximadamente el $50 \%$ del requerimiento graso diario por el feto durante el último trimestre de la gestación es provisto por la madre.

Los lípidos maternos llegan a la placenta en forma de TAG (transportados por los quilomicrones y las VLDL), así como FFA. La proporción de lípido que llega como quilomicrones y FFA, deriva en gran parte de la lipólisis del tejido adiposo almacenado dependiendo del estado de ayuno materno (55).

Los TAG no pueden cruzar la barrera placentaria, pero la superficie materna de la placenta contiene lipoproteína lipasa la cual cataliza la liberación de FFA de las lipoproteínas, especialmente de las (56) VLDL, a si mismo, FFA disociados de la albúmina sérica la cual transporta lípidos a los tejidos maternos, también proveen un importante componente del suplemento total de FFA a la placenta.
Los FFA y el glicerol pueden cruzar el borde microvelloso de las membranas placentarias, por difusión simple. En el tejido placentario, se lleva a cabo una oxidación parcial de estos, como lo confirma la presencia de peroxisomas en la placenta. Al no existir LPL en el borde fetal de la placenta, los lípidos entran a la circulación fetal como FFA y son transportados al hígado fetal unidos a la albúmina.

La placenta posee: diferentes mecanismos que potencialmente resultan en transporte preferencial de EFA y LCPUFA al feto. Los LC-PUFA probablemente deriven del hígado, ya que los hepatocitos poseen elongasas y desaturasas necesarias para producir estas moléculas utilizando EFA a la vez que poseen el mecanismo para exportarlos desde las células mediante la formación de VLDL por ejemplo. (57), siendo esta posiblemente la mayor vía de transporte de LC-PUFA derivados de la madre hacia la placenta (57).

La placenta es entonces, en gran parte un órgano fetal que presenta una composición de ácidos grasos más similar al plasma fetal que al materno $(58,59)$.

Las cantidades absolutas de ácidos grasos en plasma umbilical son significativamente mas bajos comparados con los valores maternos, debido a la menor concentración de fosfolípidos en sangre de cordón comparados con la sangre materna al nacimiento (60), concentraciones mayores de AA y DHA pueden ser explicadas por una posible síntesis de novo por el feto, pero debido a que la capacidad de la placenta y el hígado fetal para sintetizar PUFA de cadena larga (LCP de Long chain PUFA), AA y DHA (61) es muy limitada, otros mecanismos pueden ser los responsables de tal diferencia, como el transporte preferencial de LCP a través de la placenta, con una retención selectiva de AA y DHA a expensas del LA (linoleic acid) dentro de los PL (de Phospholipids ) (62), teniendo como consecuencia AA y DHA aislados dentro de una fracción lipídica que no cruza la barrera placentaria (63) y que por lo tanto permanece disponible para el desarrollo de los tejidos fetales como cerebro y retina.

\section{Perfiles lipídicos}

Debido aque la sangre de placentaes similar a la del bebé, es posible por lo tanto, establecer valores promedio de lípidos en poblaciones al nacimiento (64), como ha sido establecido el perfillípidico en nuestro medio (65), pero deben tenerse en cuenta las posibles diferencias que puedan ser ocacionadas por las diferentes patologías existentes que cambien las condiciones fisiológicas del bebé al momento del parto (66) e incluso las ocasionadas por el procedimiento en la toma de muestras (67), por eso, de ser posible todos los estudios a realizar deben presentar tendencia hacia la uniformidad en las metodologías propuestas (68).

\section{REFERENCIAS}

1. Wood P.A. Defects in mitochondrial $\beta$-Oxidation of fatty acids. Current opinion in lipidology. 1999; 10: 107-112.

2. Osario J.H. Embarazo y Metabolismo de las proteínas. Rey. Gin. Obst. 1999; 50(3): 127-132.

3. Stryer L. Fatty acid metabolism. In: Biochemistry. Freeman W.H. ed. 1997; 603-625.

4. Davison V.L. Lipids and related compounds. in: Biochemistry. Horwal Publishing. 2ed. 1994; 139-153.
5. Wanders R.J.A., Vreken P., den Boer E.J., et al. Disorders of mitochondrial fatty acid acyl-CoA $\beta$-oxidation. J. Inher. Metab. Dis. 1999; 22: 442-487.

6. Hashimoto T. Peroxisomal $\beta$-Oxidation Enzymes. Neurochem. Res. 1999; 24(4): 551-563.

7. Olsen S.F., Sorensen J.D., Scher N.J. Randomised controlled trial of effect of fish-oil supplementation on pregnancy duration. Lancet 1992; 339: 1003. 
8. Illingworth P.J., Jung R.T., Howie P.W., et al. Reduction in postprandial energy expenditure during pregnancy. British Med. J. 1987; 294: 1573.

9. Bisdec J.T., James W.P.T. Menstrual cycle hormonal changes and energy expenditure. Proceed. Nut. Soc. 1984; 43: 143A.

10. Lawrence M,. Lawrence F., Coward W.A., et al. Energy requeriments of pregnancy in the Gambia. Lancet. i: 1072.

11. Campbell-Brown M,. Hytten F .E. Nutrition. In: Clinical physiology in obstetrics. Chamberlain G., Broughton F. Eds. Blackwell Science.1998; 168-170.

12. Duam G., Vance J.E. Import of lipids into mitochondria. Prog. Lipid. Research.1997; 36: 103-130.

13. Hamilton J .A. Fatty acid transport: difficult or easy? J. Lipid Res. 1998; 39: 467-481.

14. Persson B., Hansson U. Hypoglycaemia in the pregnancy. Baillere's Clinical endocrinology and metabolismo Hypoglicaemia. Gregory J.W and AynsleyGreen A. Eds. 1993; 7(3): 731-740.

15. Hay W.W. Jr. Current topic: Metabolic interrelationships of placenta and fetus. Placenta.1995; 16: 19-30.

16. Martinez M. Tissue levels of polyunsaturated fatty acids during early human development. J. Pediatr.1992; 120: S129-S138.

17. Crawfor M.A. The role of essential fatty acids in neural development: implications for perinatal nutrition. Am. J. Clin. Nut. 1993; 57: Suppl. 793S$710 \mathrm{~S}$.

18. Campbell F.M., Gordon M.J., Dutta-Roy A.K. Preferential uptake of ling chain polyubsaturated fatty acids by isolated human placental membranes. Mol. Cell. Biochem. 1996; 155: 77-83.

19. Foreman-van Drongelen M.M., Zejdner E.E., et al. Essential fatty acid status measured in umbilical vessels walls of infants born after a multiple pregnancy. Early Hum. Dev. 1996; 18: 205-215.

20. Otto S.J., Howelingen A.C., Antal M., et al. Maternal and neonatal fatty acid status in phospholipids: an international comparative study. Eur. J. Clin. Nut. 1997; 51: 232-242.

21. Martinez M., Ballabriga A., Gil-Gibernau J.J. Lipids of the developing human retina. I. Total fatty acids, plasmalogens, and fatty acid composition of ethanolamine and choline phosphoglycerides. J. Neuroscience Research.1988; 20: 484-490.

22. Neuringer M., Connor W.E., Lin D.S., et al. Biochemical and functional effects of prenatal and postnatal w-3 fatty acid deficienciy on retina and brain in rhesus monkeys. Poceed. Nat. Acad. Sci. USA.1986; 83: 40214025.

23. Uauy R., Birch E., Birch D., et al. Visual and brain function measurements in studies of n-3 fatty acid requirements of infants. J. Pediatrics. 1992; 20: S168-S180.

24. Makrides M., Simmer K., Goggin M., et al. Erythrocyte docosahexaenoic acid correlates with the visual response of healthy, term infants. Pediatric Res. 1993; 34: 425-427.

25. Martinez M. Severe deficiency of docosahexaenoic acid in peroxisomal disorders: a defect of delta-4 desaturation? Neurology. 1990; 40: 1292-1298.

26. Martinez M., Ballabriga A. A chemical study on the development of the human forebrain and cerebellum during the bvrain "growth spurt" periodo. I. Gangliosides and plasmalogens. Brain Res. 1978; 159: 351-362.

27. Purpura D.P. Morphogenesis of the visual cortex in the preterm infant. In: Brazier M.A.B., ed. Growth and development of the brain. New2 York: Raven Press.1975: 33-49.

28. Subbiah. M.T.R. Newly recognized lipid carrier proteins in fetal life. Proceed. Soc. Exp. Biol. Med. 1991; 198: 459-499.

29. Wang Y., Kay H. H., Killam A.P. Decreased levels of polyunsaturated fatty acids in pre-eclampsia. Am. J. Obst. Gynecology.1991; 164: 812-818.

30. Vilbergson G., Samsioe G., Wennergren M., et al. Essential fatty acids in pregnancies complicated by intrauterine growth retardation. Inter. J. Gynae. Obstetrics.1991; 36: 277-286.

31. Sanjurno P., Matorras R., Perteagudo L. Influence of fatty acid fish intake during pregnancy in the polynsaturated fatty acids of erythrocyte phospolipids in the mother at labor and newborn infante. Acta Obst. Gynecol. Scand.1995; 74: 594-598.

32. Neville M.C. Adaptation of maternal lipid flux to pregnancy: Reasearch needs. Eur. J. Clin. Nut.1999; 53: Suppl 1.S120-S123.

33. Baker P., Broughton-Pipkin F. Fish-oil and pre-eclampsia. British J. Obst. Gynae.1991; 98: 499-500.

34. Andersen H.J., Andersen L.F., Fuchs A.R. Diet, pre-eclampsia and intrauterine growth retardation.Lancet. 1989; i: 1146.

35. Secher N.J., Olsen S.F. Fish-oil and pre-eclampsia (commentaries). British J. Obst. Gynae.1990; 97: 1077-1079.

36. Popesky D., Ebbeling L.R., Brown P.B., et al. Blood pressure during pregnancy in Canadian Inuit: community differences related to diet. Canadian Med. Assoc. J. 1991; 145: 445-454
37. Freinkel N. Metabolic changes in pregnancy; in Wilson, Foster, Williams' textbook of endocrinology; 7th ed. Saunders Philadelphia. 1985: 438-451.

38. Mampel T., Villarroya F., Herrera E. Hepatectomy-nephrectomy effects in the pregnant rat and fetus. Biochem. Biophys. Res. Commun. 1985; 131: 1219-1225.

39. Arguiles J., Herrera E. Lipids and lipoproteins in maternal and fetus plasma in the rat. Biol Neonate.1981; 39: 37-44.

40. Freinkel N. Effects of the conceptus on maternal metabolism during pregnancy; in Leibel, Wrenshall. On the nature and treatmen of diabetes. $1965 ; 679-691$.

41. Shambaugh G.E., Koelher R.A., Yokoo H. Fetal fuels. III. Ketone utilization by fetal hepatocyte. Am. J. Physiol. 1978; 235: E-330-E337.

42. Palacin M., Lasuncion M.A., Herrera E. Transfer from mother to fetus of $L$ alanine and glycerolin red and $48 \mathrm{~h}$-starved pregnant rats. Biochem. Soc. Trans. 1983; 11: 731-732.

43. Zorzano A., Lasuncion M.A., Herrera E. Role of the availability of substrates on hepatic and renal gluconeogenesis in the fasted pregnant rat. Metabolism, 1986; 35: 297-303.

44. Chaves J.M., Herrera E. In vitro glycerol metabolism in pregant rat. Biol. Neonate. 1980; 37: 172-179.

45. Knoop R.H., Montes A., Warth M.R. Carobhydrate and lipid metabolism in normal pregnancy. Food and nutrition board: Laboratory in dices of nutritional status in pregnancy. Nat. Acad. Sci. 1978; 35-88.

46. Knoop R.H., Boroush M.A., O'Sullivan J.B. Lipid metabolism in pregnancy. II. Postheparin lipolytic activity and hypertriglyceridemia in the pregnant Metabolism.1975; 24: 481-493.

47. Lopez-Luna P., Munoz T., Herrera E. Body fat in pregnant rats at mid gestation. Life Sci.1986; 39: 1389-1393.

48. Humphrey J.L., Childs M.T., Montes A., et al. Lipid metabolism in pregnancy VII. Kinetics of chylomycron triglyceride removalin red pregnant rat. Am. J. Physiel. 1980; 239: E81-E87.

49. Chaves J.M., Herrera E. In vitro glycerol metabolism in adipose tissue from fasted pregant rats. Biochem. Biophys. Res. Commun.1978; 85: 1299-1306.

50. asocio J.H. Es la lipoproteina lipasa (LPL) la enzima clave en el proceso de la obesidad? Lect. Nut. 1996; 14(7): 800-810.

51. Herrera D., Gomez-Coronado M.A., Lasuncion M.A. Lipid metabolism in pregnancy. Biol. Neonate. 1987; 51: 70-77.

52. Page K. Transport and metabolism of lipids in: the physiology of the human placenta. Chapter 6. UCL Press.1993; 71-76.

53. Coleman R.A. Placental metabolism and transport of lipid. Federation Proceedings. 1986; 45: 2519-2523.

54. Coleman R.A. The role of the placenta in lipid metabolism and transport. Seminars in perinatology.1989; 13: 180-191.

55. Benassayag C., Mignot T M., Haurigui M., et al. High polyunsaturated fatty acid, thromboxane A2, and alpha-fetoprotein concentrations at the human feto maternal interface. J. Lipid Res. 1997; 38: 276-286.

56. Campbell F.M., Gordon M.J., Dutta-Roy A.K. Preferential uptake of long chain polyunsaturated fatty acids by isolated human placental membranes. Mol. Cell. Biochem.1996; 155: 77-83.

57. Evain-Brion D. Maternal endocrine adaptations to placental hormaones in humans. Acta Pae. Suppl. 1999; 428: 12-16.

58. Pridjian G. Feto-Maternal interactions: placental physiology and its role as a go-between. in: Avery G.B., Fletcher M.E., Mac Donald M.G. Neonatology. 5ed. William and Wilkins.1999; 125-141.

59. Al. M.D.M., van Houwelingen A.C., Kester A.D.M., et al. Maternal essential fatty acids patterns during normal pregnancy and their relationship to the neonatal essential fatty acid status. British J. Nut. 1995; 74: 55-68.

60. Chambaz J., Ravel D., Manier M.C., et al. Essential fatty acids interconvertion in the human fetal liver. Biol. Neonate.1985; 47: 136-140.

61. Kuhn D.C., Crawford M. Placental essential fatty acid transport and prostaglandin syntehsis. Progree in lipid Research. 1986; 25: 345-353.

62. Van Houweligen AC., Puls J., Hornstra G. Essential fatty acid status during early human development. Early Human Development. 1992; 31: 97-111.

63. Leaf A.A., Leighfield M.J., Costeloe K. L., et al. Long chain polyunsaturated fatty acids and fetal growth. Early Human development. 30: 183-191.

64. Soler W., Bravo M.L., Perfillipidico en neonatos. Iatreia. 1996; 9(3): 110113

65. Osocio J.H., Quintero J.A., Osocio J.J., et al. Perfil lipidico en sangre de cordon umbilical en una poblacion Colombiana. Rev. Col. Gin. Obst. 1998; 49(2): 93-96.

66. asocio J.H., Satizabal J.M. Efecto del estres al nacimiento sobre el perfil lipidico neonatal. Rev. Memorias Congreso Nacional Soco Col. Ciencias Biológicas. 1996; 218.

67. Osorio J.H., Quintero J.A., asocio J.J., et al. Perfil lipidico neonatal vs perfillipidico placentario. Rev. Col. de Obstetricia y Ginecología. 1998; 49(3): 167-168. 